\title{
A Comparison of Nonlinear Control Performance Assessment Techniques for Nonlinear Processes
}

\author{
Wei $\mathrm{Yu}^{1 *}$, David I. Wilson ${ }^{2}$ and Brent R. Young ${ }^{3}$ \\ ${ }^{1}$ Industrial Information \& Control Centre, The University of Auckland, New Zealand \\ ${ }^{2}$ Dept. of Electrical \& Electronic Engineering, Auckland University of Technology, New \\ Zealand \\ ${ }^{3}$ Dept. of Chemical \& Materials Engineering, The University of Auckland, New Zealand
}

\begin{abstract}
Assessing the quality of industrial control loops is an important auditing task for the control engineer. However there are complications when considering the ubiquitous nonlinearities present in many industrial control loops. If one simply ignores these nonlinearities, there is the danger of over-estimating the performance of the control loop in rejecting disturbances, and thereby possibly overlooking loops that need attention. To address this problem several techniques have been recently developed to extend the control performance assessment (CPA) of single input/single output linear systems to nonlinear systems. This paper surveys these nonlinear CPA techniques and compares them using three case studies. These results can be used to guide control engineers to select the most suitable CPA techniques for their particular applications.
\end{abstract}

\section{INTRODUCTION}

Control performance assessment, or CPA, is the name given to a broad range of strategies intended to maintain operational efficiency of industrial control systems. Tasks included under the CPA umbrella include the automated diagnosis of underperforming control loops, the establishment of control benchmarks and on occasion recommending solutions. Much of the established practice in this area assumes linear plant models disturbed by Gaussian noise and has enjoyed success in the refining, petrochemicals, pulp and paper and the mineral processing industries as noted in the papers (Harris 1999; Huang and Shah 1999; Jelali 2006).

There are limits though to how far a strictly linear analysis can go when faced with the inevitable nonlinearities found in many industrial control loops. These nonlinearities could be due to the installed nature of the control valve (equal percentage, quick opening etc), the measurement transducer, or even the plant itself. Estimates of the minimum variance performance lower bound (MVPLB), which is a key component when establishing a benchmark on which to quantify the controlled performance, and the subsequent performance index using the linear CPA techniques will be distorted by these nonlinearities (Harris and Yu 2007; Prabhu 2008; Yu et al. 2010b; 2010c). For example, Yu et al. (2010c) show that one tends to overestimate the performance index for linear systems with an additive linear disturbance affected by valve stiction when using linear CPA techniques which can lead to a false sense of

* corresponding author 
security. To deal with this situation, recent research has proposed several methods to extend CPA into nonlinear systems. This paper gives an overview of these methods.

CPA techniques can be generally defined into four steps (Harris 1999; Jelali 2006): i) determining the performance benchmark; ii) detecting the poor performance loops; iii) diagnosing the reasons; vi) and finally providing the solutions. The first step, establishing the benchmark, is the key step for CPA techniques for linear systems if the process variables (PV) do not show the abnormal behavior such as oscillation and is the focus of this paper. In this paper, the nonlinear CPA techniques are used specific for the method to estimate the performance index.

Recent activity concerned with the application of CPA to nonlinear systems falls into one of two groups. The first group focused on the diagnosis of a common specific nonlinearity, such as valve stiction. Since the PV of the control loop with valve stiction problems will exhibit oscillation, the first two steps included in CPA techniques are often superfluous so control engineers can directly jump to the third step. The methods will firstly identify one of three causes of the oscillation: poor tuning, oscillatory disturbances and valve stiction. If the problem is valve stiction, the quantity measures of the valve stiction can be directly estimated from the outputs of the control loop. Further details can be found in the papers and references within them (Horch 1999; Choudhury et al. 2006; Thornhill and Horch 2007; Jelali and Huang 2009).

The second group of researchers tried to determine the performance benchmark for general nonlinear systems. Due to the complexity of the nonlinear systems, the minimum variance performance lower bound (MVPLB), or equivalently a corresponding general performance benchmark, is shown to not exist for some nonlinear systems ( $\mathrm{Yu}$ et al. 2010b). However for a restricted class of nonlinear systems, the minimum variance performance lower bound was proved to exist (Harris and $\mathrm{Yu}$ 2007). The minimum variance perfomance lower bound can be estimated using the nonlinear auto-regressive with exogenous inputs (NARX) method (Harris and $\mathrm{Yu}$ 2007), the neural network method (Zhou and Wan 2008) or a normalized performance index was proposed for run-to-run exponentially weighted moving average (EWMA) controller for nonlinear systems (Prabhu 2008). If the nonlinearity is non-differentiable (as in the case of valve stiction), the nonlinearity removal method using a some sort of semi-parametric curve such as a B-spline is proposed to estimate the MVPLB (Yu et al. 2010c) and is extended to more general block-oriented nonlinear systems ( $\mathrm{Yu}$ et al. 2010a). Important industrial nonlinearities that may affect CPA calculations are not just due to nonlinear plant dynamics, they could also arise from issues such as how the control loop is sampled and subsequently processed. The estimate of the performance index for the linear system with a sampling jitter problem was studied in the paper (Yu et al. 2011). For general nonlinear systems, a new performance index based on an ANOVA-like variance decomposition method was recently proposed (Yu et al. 2010b).

This paper summarises and compares three techniques to quantify a controller performance index intended for cases where nonlinearities are anticipated to dominate. The first method, (primarily included as a benchmark), is the standard linear approach where one simply ignores the nonlinearity. The second approach is a parametric method which was originally proposed to deal with nonlinear systems with an additive disturbances (Harris and Yu 2007; Prabhu 2008; Zhou and Wan 2008), 
and the third method is based on the technique of an ANOVA-like variance decomposition ( $\mathrm{Yu}$ et al. 2010b). In the first two methods, the performance index is calculated from the minimum variance performance lower bound (MVPLB) which in turn is estimated from a regressing linear ARMA and a nonlinear ARMA models respectively. We assume that the minimum variance performance lower bound (MVPLB) exists in these first two cases although as we shall see that assumption is not necessary for the third alternative.

The layout of the paper is as follows. In Section 2, the general nonlinear system for this paper is introduced, after which the three CPA methods developed for nonlinear systems are discussed in Section 3. In Section 4, simulation examples are used to quantify the comparison. This is followed by a discussion and conclusions highlighting both the limitations and potential of the alternative methods.

\section{PROCESS DESCRIPTION}

A general nonlinear dynamic system with stochastic disturbances shown in Figure 1 is often difficult and time consuming to identify and estimate directly. Furthermore, when faced with the task of monitoring hundreds to thousands of loops in a industrial plant, we would rather empirical identification methods that in principle we could automate.

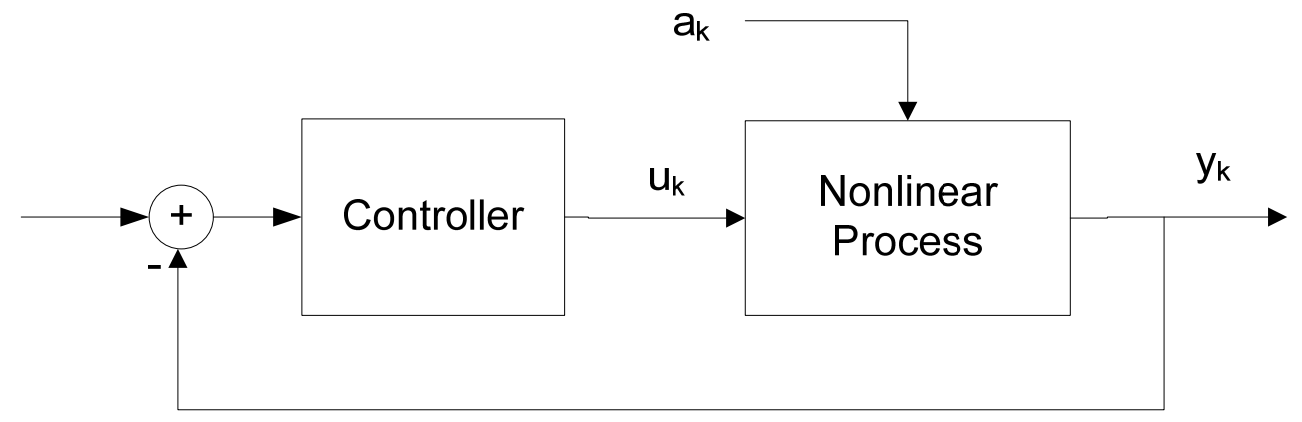

Figure 1: A general industrial nonlinear control loop

The two main reasons that the estimation is difficult in industrial circumstances is that an accurate mechanistic model of the plant dynamics and noise processes are often difficult to obtain, and that the internal state variables are usually unmeasured leaving only input-output data accessible. Consequently the only practical approach to devising and the subsequently regressing nonlinear models are those methods that are restricted to using only external data (Leontaritis and Billings 1985a; 1985b; Diaz and Desrochers 1988; Pearson and Ogunnaike 1997). The nonlinear autoregressive moving average with exogenous inputs (NARMAX) models proposed by Leontaritis and Billings (1985a; 1985b) provide a unified representation for a wide class of discrete time nonlinear dynamic / stochastic systems and hence are used for the performance assessment in this paper. In summary this paper assumes the general SISO discrete nonlinear system will be represented by a NARMAX model as,

$$
y_{k}=f\left(y_{k-1}^{*}, u_{k-b}^{*}, a_{k}^{*}\right)
$$

where $y_{k}$ is the deterministic output of the system in response to the inputs, $u_{k}$, at sample time $k$, and $a_{k}$ denotes a disturbance entering the nonlinear system. This noise 
term is assumed to be an independent identically distributed (i.i.d.) random variable with a mean and constant variance $\sigma_{a}^{2}$. The integer $b$ represents the number of whole periods of delay in the system and is the number of sample intervals that elapse between making a change in the process input and first observing its effect. The superscript * is used to represent the vector collecting the immediate past values i.e. $y_{k-1}^{*}=\left[y_{k-1}, \ldots, y_{k-n_{y}}\right]$ where $n_{y}$ is the length of the memory.

Given that the relation $f(\cdot)$ is often complex, one is forced to approximate the nonlinearity with a simpler generic function, typically polynomials consisting of summations of terms involving $y_{k-i}, y_{k-i} y_{k-j}, \ldots, u_{k-b-i}, u_{k-b-i} u_{k-b-j}, \ldots, a_{k-i}, a_{k-i} a_{k-j}$ and cross terms i.e. $y_{k-i} u_{k-b-j}, y_{k-i} a_{k-j}$ and $u_{k-b-i} a_{k-j}$. The flexible and popular Hammerstein and Weiner systems are included in this framework (Pearson 1999).

\section{TECHNIQUES OF ESTIMATING THE PERFORMANCE INDEX FOR NONLINEAR PROCESSES}

Before we describe methods for nonlinear systems, we will revise the CPA for linear systems. A linear system disturbed by noise is

$$
y_{k}=\frac{B\left(q^{-1}\right)}{A\left(q^{-1}\right)} u_{k-b}+d_{k}
$$

where $A\left(q^{-1}\right)$ and $B\left(q^{-1}\right)$ are polynomials in the backshift operator $q^{-1}$, defined such that $q^{-1} y_{\mathrm{k}}=y_{k-1}$. The disturbance $d_{k}$ is modeled as the output of a linear filter driven by white noise. This type of disturbance is conveniently represented by an Autoregressive-Moving-Average (ARMA) time series model of the form:

$$
d_{k}=\frac{C\left(q^{-1}\right)}{D\left(q^{-1}\right)} a_{k}
$$

where $C\left(q^{-1}\right)$ and $D\left(q^{-1}\right)$ are monic and stable polynomials, and $a_{k}$ denotes a disturbance entering the nonlinear system with properties as previously mentioned. This disturbance model will be used for all the disturbances studied in this paper

The performance index proposed for SISO linear systems proposed by Harris (1989) was based on the concept of minimum variance control (Astrom 1970). This performance index, often termed the Harris Index, $\eta$, was defined as the ratio of the best achievable variance to the actual variance of the controlled variable under assessment,

$$
\eta=\frac{\sigma_{M V}^{2}}{\sigma_{y}^{2}}
$$

where $\sigma_{M V}^{2}$ denotes the minimum variance performance lower bound and $\sigma_{y}^{2}$ denotes the actual output variance of the control loop under consideration.

What made this concept useful was the insight that $\eta$ can be estimated directly from routine operating data by fitting the controlled variable to an ARIMA time series model (Harris 1989). In the following three sections, three different techniques based on this performance index will be discussed in detail. 


\subsection{Applying a linear CPA technique for nonlinear systems}

For nonlinear systems described in Equation (1), we can simply use a linear ARMA model to approximate these nonlinear systems as,

$$
\begin{aligned}
y_{k} & =f\left(y_{k-1}^{*}, u_{k-b}^{*}, a_{k}^{*}\right) \\
& \approx \frac{B_{1}\left(q^{-1}\right)}{A_{1}\left(q^{-1}\right)} u_{k-b}+\frac{C_{1}\left(q^{-1}\right)}{D_{1}\left(q^{-1}\right)} a_{k}
\end{aligned}
$$

The approximate performance index can be calculated as,

$$
\hat{\eta}=\frac{\hat{\sigma}_{M V}^{2}}{\sigma_{y}^{2}}
$$

where $\hat{\sigma}_{M V}^{2}$ denotes the minimum variance performance lower bound which is estimated from the approximate linear ARMA model in Equation (5).

$$
\hat{\sigma}_{M V}^{2}=\left(1+\psi_{1}^{2}+\ldots+\psi_{b-1}^{2}\right) \hat{\sigma}_{a}^{2}
$$

and $1, \psi_{1}, \ldots, \psi_{b-1}$ are the first $b$ impulse response weights of the disturbance model $C_{1}\left(q^{-1}\right) / D_{1}\left(q^{-1}\right)$ in Equation (5). However due to the fact that we have simply ignored the nonlinearity, it is shown that this estimate will be biased and will overestimate the true MVPLB for a class of nonlinear systems (Yu et al. 2010c). It is interesting to note that had a linearised analysis under-estimated the performance benchmark, we could have safely used this procedure while acknowledging that we may be doing extra work investigating loops that are reported as poor performing when in fact they are not. However that is not the case. Since the linearised analysis over-estimates the performance, then we have now a false sense of security and potentially ignore control loops that should be further investigated.

\subsection{A parametric CPA method for the nonlinear process}

A better approximation of the nonlinear process in Equation (1) than the linear approximation in Equation (5) would naturally be a combination of a nonlinear dynamic plant model plus an additive linear disturbance model (such as the disturbance model in Equation (3)). Such a model can be expressed as,

$$
\begin{aligned}
y_{k} & =f\left(y_{k-1}^{*}, u_{k-b}^{*}, a_{k}^{*}\right) \\
& \approx N\left(y_{k-1}^{*}, u_{k-b}^{*}\right)+\frac{C_{2}\left(q^{-1}\right)}{D_{2}\left(q^{-1}\right)} a_{k}
\end{aligned}
$$

where $N(\cdot)$ is a generic nonlinear function.

If a nonlinear dynamic/stochastic system can be adequately modeled by Equation (8), Harris and Yu (2007) proved that a minimum variance feedback invariant exists and that the minimum variance performance lower bound can still be estimated from routine operating data. The minimum variance performance lower bound, $\hat{\sigma}_{M V}^{2}$, can also be written the similar form to Equation (7), but the coefficients, $1, \psi_{1}, \ldots, \psi_{b-1}$, are now the first $b$ impulse response weights of the disturbance model $C_{2}\left(q^{-1}\right) / D_{2}\left(q^{-1}\right)$ 
in Equation (8). The $\hat{\sigma}_{a}^{2}$ is the variance estimate of noise sequence $a_{k}$ and can be directly estimated by fitting a nonlinear ARMA model corresponding to the $b$-step ahead prediction of outputs. This derivation can be found in the paper (Harris and $\mathrm{Yu}$ 2007).

When the nonlinear function $N(\cdot)$ is non-differentiable as in the case of valve stiction or sample jitter, a semi-parametric CPA method for nonlinear systems was proposed to find the MVPLB for linear systems by Yu et al. (2008; 2009). The approach was first to remove the nonlinearity in the observable time series $y$ using smoothing B-splines where the degree of smoothing, (tolerance, $\lambda$ ), is adjusted iteratively. The procedure consists of two parts: i) A non-parametric B-spline is used to fit the nonlinearity from the output and ii) a linear ARMA model is used to fit the residuals between the output and B-spline. Consequently the minimum variance performance lower bound can be estimated given the regressed ARMA model to the now linear data series. The extent of smoothing to just remove the nonlinearity is established by checking the Gaussianity and linearity of the residuals using the nonparametric Hinich test (Hinich 1982). The application of this linearity test on the diagnosis and detection of valve stiction was reported in (Choudhury et al. 2006).

\subsection{ANOVA-based CPA method for nonlinear processes}

The MVLPB-based performance indices described in Sections 3.1 and 3.2 exhibit significant theoretical difficulties when applied to general nonlinearities such as NARMAX models. Quite apart from the fact that the MVPLB may be difficult to estimate, or even may not exist, the idea of variance decomposition using the impulse response function and the concept of feedback invariance is not necessarily valid for nonlinear systems.

To address these more general nonlinear cases, we take a more fundamental statistical approach by looking at variance components of the data. The ANOVA-like variance decomposition method was used to provide the variance analysis for nonlinear systems with multi-disturbance sources (Harris and Yu 2010; 2011). Some previous work using a variance decomposition for linear systems in a feedback/feedfoward SISO configuration, in a MIMO linear system, and in a SISO cascaded systems is given in (??). However instead of the different disturbance sources, the ANOVA-like variance decomposition will be used with differing time horizons for the SISO nonlinear system with one disturbance source as developed in (Yu et al. 2010b).

We can take an analogous approach where we separate the disturbances entering the system described in Equation (1) after some nominal start time 0, say $\left[a_{k+b}, a_{k+b}\right.$ $\left.1, \ldots, a_{0}\right]$, into two groups shown in Figure 2. The first group includes all the disturbances entering the system after the current time $k$, namely $A_{1}=\left[a_{k+b}, a_{k+b-l}, \ldots\right.$ ,$\left.a_{k+1}\right]$ and the remaining group which is all the disturbances entering the system from the start time, 0 , to the current time $k$, namely $A_{2}=\left[a_{k}, a_{k-1}, \ldots, a_{0}\right]$. 


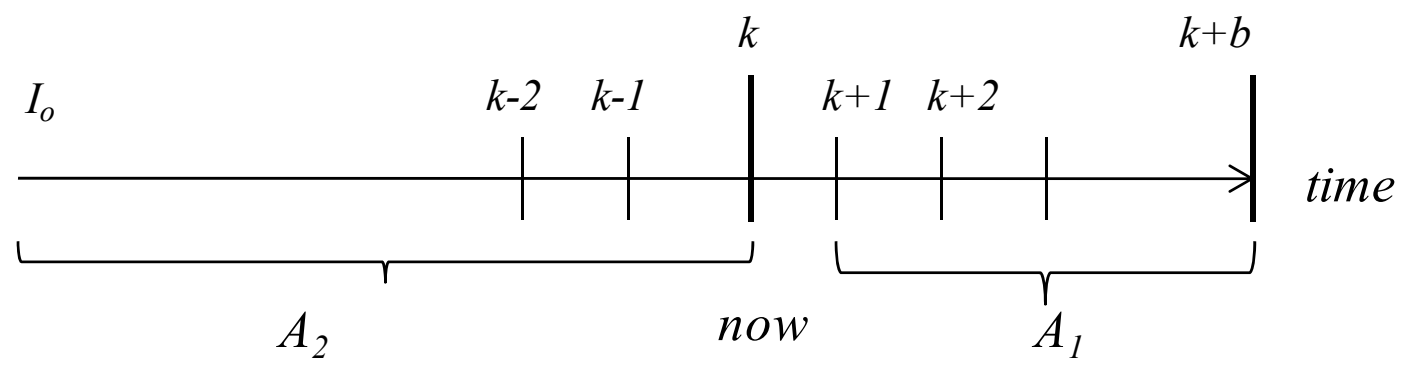

Figure 2: Dividing the disturbance sequences into two groups, $A_{1}$ and $A_{2}$, separated in time

We are interested in determining the sensitivity of output $y_{k+b}$ in Equation (1) to variations of the two vector series $A_{1}$ and $A_{2}$. Since the future behavior of $y_{k+b}$ is dependent on initial conditions due to the nonlinearity, we can use the well-known variance decomposition theorem (Parzen 1962) to deal with the effect of the unknown initial conditions. The variance of $y_{k+b}$ can be decomposed into two terms:

$$
V\left[y_{k+b}\right]=E_{I_{0}}\left[V_{A}\left[y_{k+b} \mid I_{0}\right]\right]+V_{I_{0}}\left[E_{A}\left[Y_{k+b} \mid I_{0}\right]\right]
$$

where $A=\left[A_{1}, A_{2}\right]$ denotes all disturbances entering the system from time 0 to time $\mathrm{k}$, $E_{I 0}[\cdot]$ denotes the expectation of [.] with respect to $I_{0}$ and $V_{I 0}[\cdot]$ denotes the variance of [.] with respect to $\mathrm{I}_{0}$. Given that Equation (9) is the sum of positive numbers, it follows that $V\left[y_{k+b}\right] \geq E_{I_{0}}\left[V_{A}\left[y_{k+b} \mid I_{0}\right]\right]$.

The first term on the right hand side of Equation (9) is the fractional contribution to the variance of $y$ from the disturbance signal and the interaction between the disturbance and the initial condition. The second term is the fractional contribution to the output solely due to the uncertainties in the initial condition.

The conditional variance given the initial condition $I_{0}, V_{A}\left[y_{k+b} \mid I_{0}\right]$, can be decomposed directly using the ANOVA-like decomposition method as:

$$
\begin{aligned}
& V_{A} \mid I_{0}=V_{A}\left[y_{k+b} \mid I_{0}\right] \\
& =V_{1}\left|I_{0}+V_{2}\right| I_{0}+V_{12} \mid I_{0}
\end{aligned}
$$

where the terms $V_{1}, V_{2}$ and $V_{12}$ are

$$
\begin{aligned}
& V_{1} \mid I_{0}=V_{A_{1}}\left[E_{A_{2}}\left[y_{k+b} \mid\left(A_{1}, I_{0}\right)\right]\right] \\
& V_{2} \mid I_{0}=V_{A_{2}}\left[E_{A_{1}}\left[y_{k+b} \mid\left(A_{2}, I_{0}\right)\right]\right] \\
& V_{12}\left|I_{0}=V_{A}\left[E_{A}\left[y_{k+b} \mid\left(A, I_{0}\right)\right]\right]-V_{1}\right| I_{0}-V_{2} \mid I_{0} \\
& \quad=V_{A}\left|I_{0}-V_{1}\right| I_{0}-V_{2} \mid I_{0}
\end{aligned}
$$

The variance decomposition with consideration to the initial condition can be obtained by simply calculating the expectation of the conditional variance decomposition in Equation (11) with respect to the initial condition $\mathrm{I}_{0}$. This procedure can be omitted if the initial condition has, (or can be approximately assumed to have), a linear relationship with the output $y_{k+b}$. The variance decomposition can be calculated with the results of the conditional variance decomposition in Equation (11) based on the mean values of initial condition. Further information about this topic can be found in the paper (Yu and Harris 2009). 
$E_{I 0}\left[V_{l} \mid I_{0}\right]$ denotes the main effect of $A_{1}$ on the $V\left[y_{k+b}\right]$ and $E_{I 0}\left[V_{12} \mid I_{0}\right]$ is the interaction contributing to the $V\left[y_{k+b}\right]$ that is not accounted for in the main effects of $A_{1}$ and $A_{2}$. Consequently we propose a suitable performance index as,

$$
\tau=\frac{E_{I_{0}}\left[V_{1} \mid I_{0}\right]}{\sigma_{y+b}^{2}}
$$

While this index is applicable for any nonlinearity, the index has little worth for those processes that are strongly non-ergodic where the second term in Equation (9) dominates. However such cases are more pathological than common in industry.

This performance index shares many of the key properties of the Harris index. . The performance index is strictly bounded between zero and one. If $\tau$ reaches 1 , it means that the variance of the outputs is contributed mostly by $A_{1}$, so the controller approaches minimum variance control. Furthermore the partial variance $E_{I 0}\left[V_{l} \mid I_{0}\right]$ is equal to the minimum variance performance lower bound for some nonlinear systems such as those discussed in the paper (Harris and Yu 2007) and the partial variance $E_{I 0}\left[V_{l} \mid I_{0}\right]$ is equal to the minimum variance performance lower bound if the closed loop system is linear and stationary. Consequently the performance index in Equation (12), $\tau$, plays a very similar role to that of the Harris index, $\eta$. However unlike $\eta, \tau$ may depend on the controller, the initial condition and the length of time $k+b$.

The practical computation of the performance index in Equation (12) requires two steps. First one must estimate the closed loop model defined in Equation (1), and then use a Monte-Carlo strategy to estimate the performance index in Equation (12).

For the first identification step several strategies have been proposed such as Orthogonal Least Squares (OLS) methods (Chen et al. 1989), Fast Orthogonal Search (FOS) methods (Korenberg 1988), and approximations based on Artificial Neural Network (ANN) models (Chen and Billings 1992). In this paper, we will use the OLS method to estimate the parameters of the nonlinear systems in Equation (1).

Since the disturbance term in Equation (1) is generally unmeasured, the identification be recursive. The identification procedures first sets the initial sequence $a_{\mathrm{k}}$ by fitting a linear model or setting the $a_{\mathrm{k}}$ to zero then identifies the NARMA model, and finally replaces the initial sequence $a_{\mathrm{k}}$ by the prediction errors or residuals. The identification and replacement steps are repeateduntil a certain identification quality is achieved as quantified by Akaike's Information Criterion (AIC). The detailed iteration procedures can be found in the paper (Chen et al. 1989).

For the second step, performance index calculation, since it is impossible to obtain the analytical solution of the variance decomposition in Equation (10), a Monte Carlo (MC) method is used to estimate the results. However MC procedures are computationally demanding especially for the high dimensional cases (Rabitz et al. 1999). The Fourier amplitude sensitivity test (FAST) method (Cukier et al. 1978; Saltelli 2002) and Sobol's method (Sobol' 1993) have been developed to reduce the intensive computation by selecting the samples more efficiently.

\subsection{Comparison of the three CPA methods intended for nonlinear systems}


For brevity, we will use method 1 to represent linear CPA technique in Section Error! Reference source not found., method 2 to denote parametric nonlinear CPA technique in Section 3.2 and method 3 to denote the ANOVA-like nonlinear CPA technique in 3.3.

General speaking, the method 1 will provide overestimates for most nonlinear systems. On the plus side, the implementation of this linear method is simple, cheap and the results and underlying algorithm intuitive to operators and control engineers. However the reliability of the performance index estimate derived from method one will significantly depend on the nonlinearity of the nonlinear systems. Before using this CPA method blindly, it is necessary to check the extent of the nonlinearity. If the nonlinearity is significant, then this CPA method should not be used as a reliable tool.

Method 2 will provide a better estimate of the performance index than the method 1 , since the closer approximation of the nonlinear model structure is used for this method. However a key assumption of this method is that the disturbance is added after the dynamic process will limit the applicability of this approach. This is similar to the output-error $(\mathrm{OE})$ versus ARX model alternatives.

Since methods 1 and 2 are both based on the minimum variance performance lower bound, it follows that the existence of the control invariant is automatically assumed which may not be always applicable for general nonlinear systems. Method 3, the ANOVA-like CPA technique, can be used to assess the control loop performance in the cases where the control invariant does not exist, but the method does demand intensive computation.

\section{SIMULATIONS}

Many industrial plants can be adequately modeled by nonlinear block-oriented models which consist of the interconnection of a linear time invariant (LTI) systems with static, or memoryless, nonlinearities. This class includes Hammerstein models, Wiener models and combinations of the two (Pearson 1999). Such block-oriented nonlinear descriptions are very useful for modeling input nonlinearities such as equal percentage valve characteristics, quantization due to pulse-width modulated controllers, or output nonlinearities such as thermocouple or thyristor transducer calibration curves, and/or the digital quantization due to a crude A/D converter.

In this paper, we will use the Hammerstein-Wiener (HW) model shown in Figure 3 to demonstrate the three CPA methods: linear CPA (method 1), parametric CPA (method 2) and an ANOVA-like CPA (method 3) methods discussed in Section 3.

A typical HW model can be expressed as,

$$
\begin{aligned}
& y_{k}=N_{2}\left(x_{k}\right)+j \cdot d_{k} \\
& x_{k}=w_{k}+(1-j) \cdot d_{k} \quad j \in(0,1) \\
& w_{k}=\frac{B\left(q^{-1}\right)}{A\left(q^{-1}\right)} q^{-b} v_{k} \\
& v_{k}=N_{1}\left(u_{k}\right)
\end{aligned}
$$

where $A\left(q^{-1}\right)$ and $B\left(q^{-1}\right)$ are backward shift polynomials as previously defined, $b$ is the time delay of the system, $u_{k}$ and $y_{k}$ are the process input and output respectively, and the internal signals $v_{k}, w_{k}$ and $x_{k}$ are assumed unmeasured. The functions $N_{1}$ and $N_{2}$ 
represent static nonlinearities. The position where the disturbance enters the system is controlled by the switch $j$. It is important to note that only the signals $u_{k}$ and $y_{k}$ are observable under normal operational conditions.

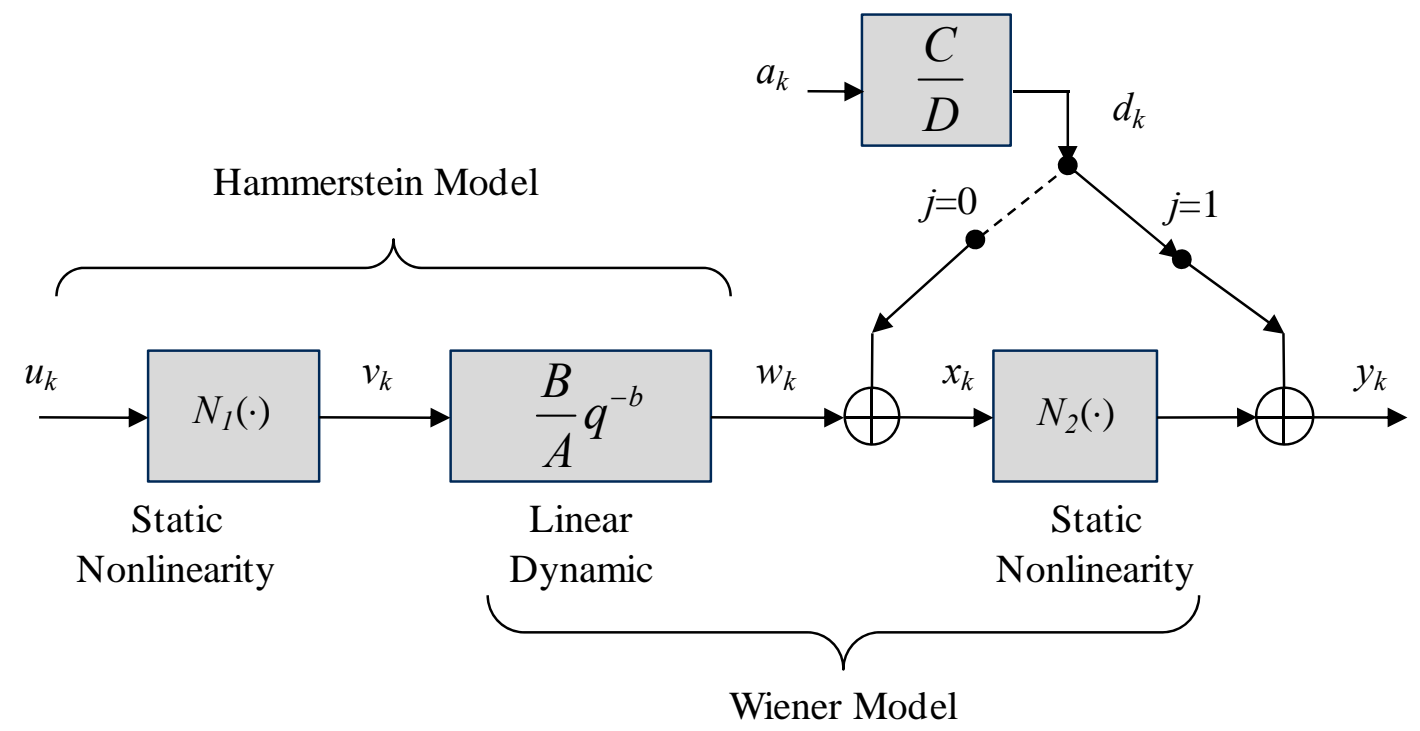

Figure 3: A diagram of Hammerstein-Wiener model used for these simulations.

As indicated in Figure 3, we consider two different locations where the disturbance can enter the system. The first location $(j=1)$ is immediately downstream of the static nonlinearity $N_{2}$, and the second location $(j=0)$ is upstream of the static nonlinearity $N_{2}$. These two different locations will be studied in cases 2 and 3 respectively.

\subsection{Case study one}

The first simulation model is a Hammerstein model following Equation (13) with a static nonlinearity depicted in the left position in

Figure 4, as

$$
N_{1}\left(u_{k}\right)=1.2 u_{k} e^{-2 u_{k}}+0.1 u_{k}
$$

And with the linear plant model and a PI controller,

$$
\begin{aligned}
& \frac{B\left(q^{-1}\right)}{A\left(q^{-1}\right)} q^{-b}=\frac{1-0.5 q^{-1}}{1-1.5 q^{-1}+0.7 q^{-2}} q^{-3} \\
& G c=\frac{0.2-0.15 q^{-1}}{1-q^{-1}}
\end{aligned}
$$

and an additive ARMA disturbance

$$
d_{k}=\frac{1}{1-0.8 q^{-1}} a_{k}, \quad \sigma_{a}^{2}=0.1
$$

The right side subplot in

Figure $\mathbf{4}$ compares the results of the estimates of the performance index obtained via the linear, nonlinear ARMA method, and the NOVA-like method in Section 3 for this Hammerstein model structure. To obtain statistically reliable results, 1000 closed loop data points were used to assess the controlled performance, and this procedure was then repeated 500 times. Testing the algorithm over a range of cases indicated that 
500 times was a reasonable balance between accuracy and computation load. The true value of performance index, $\eta$, is given by the dashed horizontal line in the right sub-plot in

Figure 4.
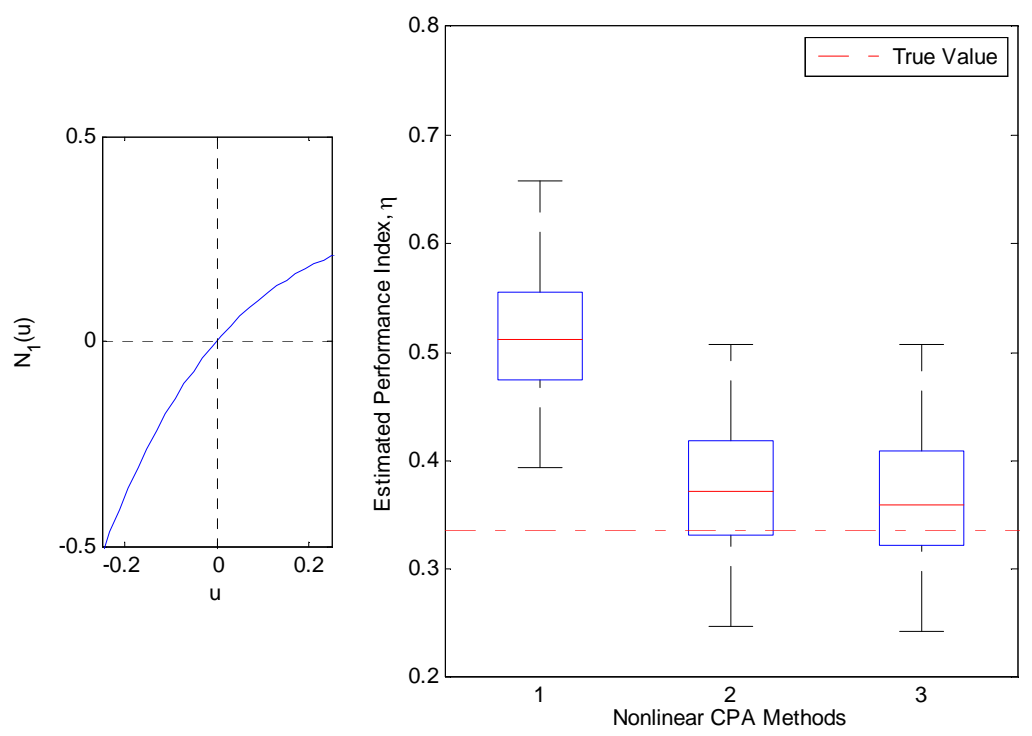

Figure 4: The nonlinearity of interest $N_{1}(u)$, (left plot), and the estimates of the performance index for Case study one, (right plot).

From

Figure 4, one can see that the estimates from methods 2 and 3 provide a reasonably reliable estimate of $\eta$ although the mean values are around $10 \%$ above the true performance index. There is no significant difference between these two nonlinear CPA methods since the nonlinear model structure is, perhaps unreasonably, correctly chosen in the case for method 2. However the largest bias can be observed for the performance index estimate from CPA method 1, (the linear CPA technique), where the deviation to the true performance index is over $50 \%$. This is a direct consequence of neglecting the nonlinearity.

As mentioned in the previous section, CPA method 2 will overestimate the performance index given a mismatch between the model structure assumed for CPA method 2 and the true dynamic/stochastic process. This phenomenon will be demonstrated in the following simulations.

\subsection{Case study two}

For this case study, we have chosen a HW model employing both $N_{1}$ and $N_{2}$. The linear process is same as the Case study one with a feedback PI controller,

$$
G_{c}=\frac{0.12-0.08 q^{-1}}{1-q^{-1}}
$$

and with a non-differentiable coulombic and viscous friction nonlinearity $N_{1}$, ,

$$
N_{1}(u)=\operatorname{sign}(u)(|u|+0.07)
$$


where 0.07 is the offset, and a smooth downstream nonlinear element $N_{2}$ as a third-order polynomial,

$$
N_{2}(x)=x+0.25 x^{2}+0.125 x^{3}
$$

The additive disturbance model used in Case study one is also used for this simulation (but the location where the disturbance enters the system is now after static nonlinearity $N_{2}$ ). The $a_{k}$ is a sequence of i.i.d. Gaussian random variable with zero mean and constant variance $\sigma^{2}{ }_{a}=0.1$. The same procedures of estimating the performance index using the three considered methods are repeated here, and the estimates are plotted in Figure 5. For convenience, the static nonlinearities $N_{l}$ and $N_{2}$ are also shown on the left side column in Figure 5.
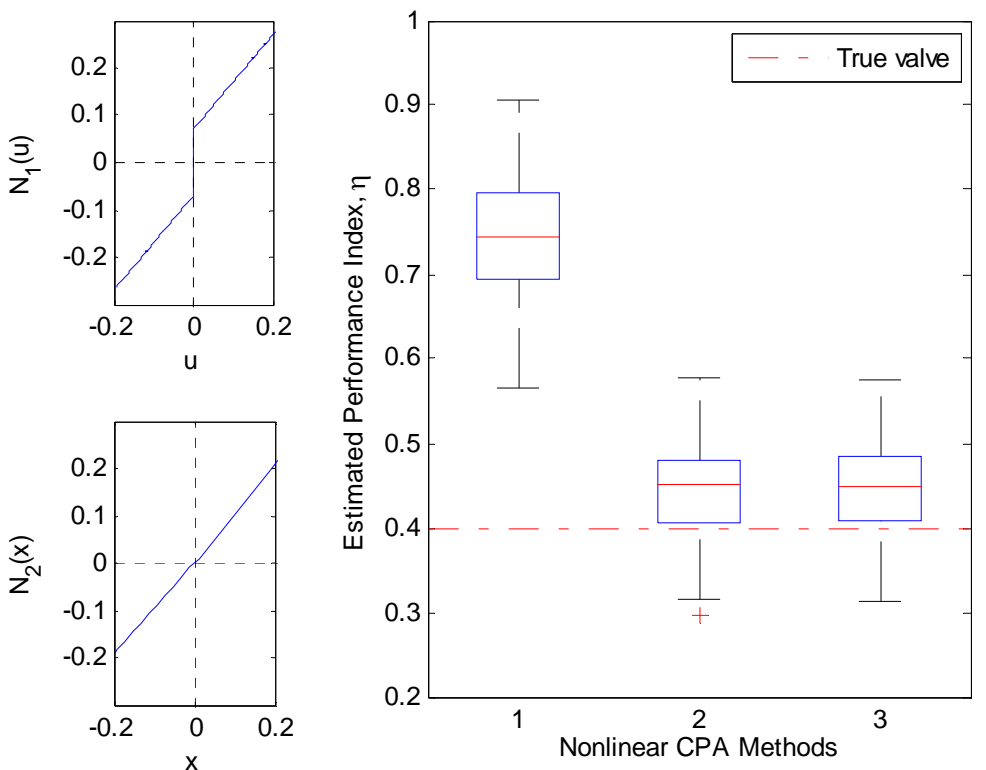

Figure 5: The two static nonlinear elements (left-hand plots) and estimates of the performance for Case study two.

The estimates from the nonlinear CPA methods 2 and 3 still provide good results with the mean values around $12 \%$ above the true performance index. Again the largest bias occurs when using the linear approximation where the deviation to the true performance index is over $86 \%$. The deviations of estimates are slightly larger than the results in the case study one due to the fact that the first nonlinear element is nondifferentiable.

\subsection{Case study three}

In this simulation, the only difference to Case study 2 is the position where the disturbance enters the control loop. Now the disturbance, $d_{k}$, is placed before the output static nonlinearity block, $N_{2}$, as opposed to the usual assumption where the disturbance is assumed additive to the output after the nonlinearity as in the Case studies one and two. In some instances this early entrance of the disturbance signal is more realistic from a process operation point of view given that it is the transducer that often provides the nonlinear behaviour as discussed in the paper (Hagenblad et al. 2008). The estimates of the performance indices are plotted in Figure 6. Notice that the true value in Figure 6 is not the minimum variance performance lower bound, it is 
a relative minimum variance performance bound and readers can find the detailed information in the paper (Yu et al. 2010b).

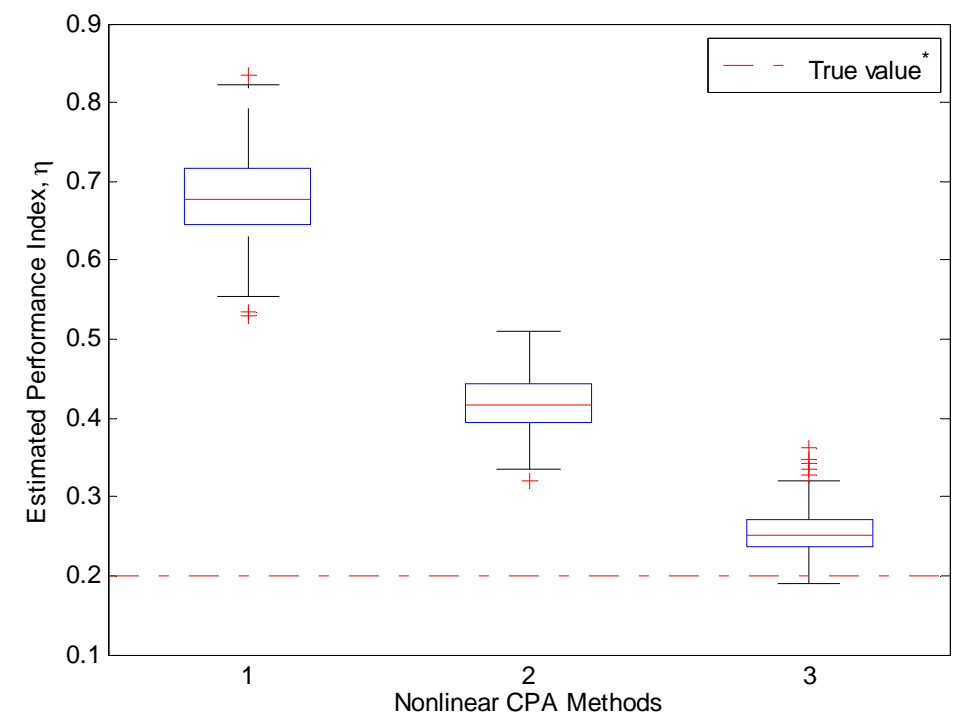

Figure 6: Estimates of the performance index for Case study three

For this case study, both the linear CPA method 1 and the non-parametric method 2 deliver large biases since the wrong model structure is used to estimate the MVPLB. However the estimate from method three provides a reliable result which, in this case, indicates that the control loop needs immediate treatment. This example highlights the danger of simply relying on a linear CPA analysis since method 1 indicates that the loop performance is satisfactory, while in reality, and as highlighted by method 3 , that is in fact not the case, and that the loop does not do an adequate job in disturbance rejection. Also notice that the location where the disturbance enters the dynamic system will dramatically affect the controller performance, since the true values of the performance indices are much smaller than the case study one.

Although the results from the ANOVA-based method (method 3) are preferred in cases, the drawback of this method is that it requires substantially more computation compared to the linear case as indicated by the computation time figures given in Table 1. Under industrial conditions it may well be that the faster computation of the nonlinear ARMAX method may more than compensate for the small bias in the estimates of the performance indices.

Table 1: Summarizing the abilities of the three methods

\begin{tabular}{|l|c|c|c|}
\hline \multirow{2}{*}{ Case Study } & \multicolumn{3}{|c|}{ Alternative CPA methods } \\
\cline { 2 - 4 } & Linear & Nonlinear & ANOVA-like \\
\hline One & $\times$ & $\checkmark$ & $\checkmark$ \\
\hline Two & $\times$ & $\checkmark$ & $\checkmark$ \\
\hline Three & $\times$ & $\times$ & $\checkmark$ \\
\hline $\begin{array}{l}\text { Computational Time } \\
\text { (Relative to the linear case) }\end{array}$ & 1 & 2 & 15 \\
\hline Average bias (\%) & 45 & 23 & 11 \\
\hline
\end{tabular}




\section{CONCLUSIONS}

This paper compared three techniques for estimating the performance index for nonlinear systems: a linear technique, a parametric method and an ANOVA-based method. Not unexpectedly the linear CPA strategy delivered results with a significant bias leading to an overoptimistic assessment of the controller's performance and this conclusion is also reflected by simulations in all three cases. The parametric method gave satisfactory results for the case when the disturbance was added directly to the output, but failed in the case where the disturbance entered the system upstream of the trailing nonlinearity. For this latter case, only the ANOVA-based strategy could deliver reliable results in all cases, but that came at a cost of substantial computation compared to the alternatives.

\section{ACKNOWLEDGMENTS}

The authors gratefully acknowledge the financial support to this project from the Industrial Information and Control Centre $\left(\mathrm{I}^{2} \mathrm{C}^{2}\right)$, Faculty of Engineering, The University of Auckland, New Zealand.

\section{REFERENCE}

Astrom, K. J., "Introduction to Stochastic Control Theory",Academic Press, New York (1970).

Chen, S. and S. A. Billings, "Neural networks for nonlinear dynamic system modeling and identification", International Journal of Control 56 (2), 319-346 (1992).

Chen, S., S. A. Billings and W. Luo, "Orthogonal least squares methods and their application to nonlinear system identification", International Journal of Control 50 (5), 1873-1896 (1989).

Choudhury, M. A. A. S., S. L. Shah, N. F. Thornhill and D. S. Shook, "Automatic detection and quantification of stiction in control valves", Control Engineering Practice 14, 1395-1412 (2006).

Cukier, R. I., H. B. Levine and K. E. Shuler, "Nonlinear sensitivity analysis of multiparameter model systems", Journal of Computational Physics 26, 1-42 (1978).

Diaz, H. and A. A. Desrochers, "Modeling of nonlinear discrete-time systems from input-output data", Automatica 5, 629-641 (1988).

Hagenblad, A., L. Ljung and A. Wills, "Maximum likelihood identification of Wiener models", Automatica 44, 2697-2705 (2008).

Harris, T. J., "Assessment of control loop performance", Canadian Journal of Chemical Engineering 67, 856-861 (1989).

Harris, T. J., "A review of performance monitoring and assessment techniques for univariate and multivariate control systems", Journal of Process Control 9 (1), 1-17 (1999).

Harris, T. J. and W. Yu, "Controller assessment for a class of nonlinear systems", Journal of Process Control 17, 607-619 (2007).

Harris, T. J. and W. Yu, "Variance decompositions of nonlinear dynamic stochastic systems", Journal of Process Control 20 (2), 195-205 (2010).

Harris, T. J. and W. Yu, "Variance decompositions of nonlinear time series using stochastic simulation and sensitivity analysis", Statistics and Computing, online April, 2011 (2011).

Hinich, M. J., "Testing for Gaussianity and linearity of a stationary time series", Journal of Time Series Analysis 3 (13), 169-176 (1982). 
Horch, A., "A simple method for detection of stiction in control valves", Control Engineering Practice 7, 1221-1231 (1999).

Huang, B. and S. L. Shah, "Performance assessment of control loops: theory and applications", Springer, London, Great Britain (1999).

Jelali, M., "An overview of control performance assessment technology and industrial applications", Control Engineering Practice 14 (5), 441-466 (2006).

Jelali, M. and B. Huang, "Dection and diagnosis of stiction in control loops; state of the art and advanced methods", Springer, London, Great Britain (2009).

Korenberg, M. J., "Identifying nonlinear difference equation and functional expansion representations: fast orthogonal algorithm", Annals of Biomedical Engineering 16, 123-142 (1988).

Leontaritis, I. J. and S. A. Billings, "Input-output parametric models for nonlinear systems, part I: deterministic nonlinear systems", International Journal of Control 41 (4), 30-328 (1985a).

Leontaritis, I. J. and S. A. Billings, "Input-output parametric models for nonlinear systems, part II: stochastic nonlinear system", International Journal of Control 41 (4), 329-344 (1985b).

Parzen, E., "Stochastic Processes", Holden Day, San Francisco (1962).

Pearson, R. K., "Discrete-Time Dynamic Models", Oxford University Press, New York (1999).

Pearson, R. K. and B. A. Ogunnaike, "Nonlinear Process Identification", Nonlinear Process Control. M. A. Henson and D. E. Seborg. Upper Saddle River, N.J., Prentice Hall: pp. 11-102 (1997).

Prabhu, A. V. (2008). Performance monitoring of run-to-run control systems used in semiconductro manufacturing. Dept. of Chemical Engineering. Austin, USA, The University of Texas.

Rabitz, H., O. F. Alis, J. Shorter and K. Shim, "Efficient input-output model representations", Computer Physics Communications 117, 11-20 (1999).

Saltelli, A., "Sensititvity analysis for importance assessment", Risk Analysis 22 (3), 579-590 (2002).

Sobol', I. M., "Sensitivity estimates for nonlinear mathematical models", Matematicheskoe Modelirovanie, 2, 112-118 (in Russian), translated in Mathematical Modeling and Computational Experiments 1, 407-414 (1993).

Thornhill, N. F. and A. Horch, "Advances and new directions in plant-wide disturbance detection and diagnosis", Control Engineering Practice 15, 11961206 (2007).

$\mathrm{Yu}, \mathrm{W}$. and T. J. Harris, "Parameter uncrertainty effects on variance-based sensitivity analysis", Reliability Engineering and System Safety 94 (2), 596-603 (2009).

$\mathrm{Yu}$, W., D. I. Wilson and B. R. Young, "Control performance assessment in the presence of valve stiction", in: "The Eleventh IASTED International Conference on Intelligent Systems and Control, ISC 2008", Orlando, Florida, USA, November 16--18, 2008 pp. 379-384 (2008).

$\mathrm{Yu}$, W., D. I. Wilson and B. R. Young, "Eliminating valve stiction nonlinearities for control performance assessment", in: "International symposium on advanced control of chemical processes", Istanbul, Turkey, July 5-7, 2009, pp. 526-531 (2009).

Yu, W., D. I. Wilson and B. R. Young, "Control Performance Assessment for Blockoriented Nonlinear Systems", in: "8th IEEE International Conference on Control \& Automation ICCA'10", Xiamen, China, June 9-11, 2010, pp. 11511156 (2010a). 
Yu, W., D. I. Wilson and B. R. Young, "Control Performance Assessment for Nonlinear Systems", Journal of Process Control 20 (10), 754-761 (2010b).

Yu, W., D. I. Wilson and B. R. Young, "Nonlinear Control Performance Assessment in the Presence of Valve Stiction", Journal of Process Control 20 (6), 754-761 (2010c).

Yu, W., D. I. Wilson and B. R. Young, "Control performance assessment in the presence of sampling jitter", Chemical Engineering Research and Design, online July, 2011 (2011).

Zhou, Y. F. and F. Wan, "A neural netwark approach to control performance assessment", International Journal of Intelligent Computing and Cybernetics 1 (4), 1617-1633 (2008). 